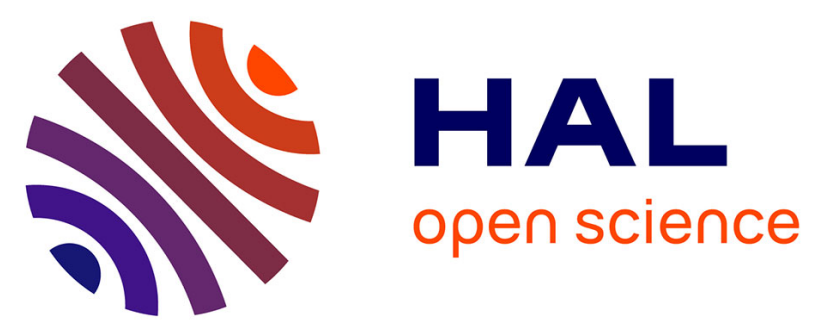

\title{
A Bayesian approach for the optimal integration of renewable energy sources in distribution networks over multi-year horizons
}

\author{
Bruno Barracosa, Juliette Morin, Héloïse Dutrieux Baraffe, Julien Bect, \\ Emmanuel Vazquez, Josselin Fournel
}

\section{To cite this version:}

Bruno Barracosa, Juliette Morin, Héloïse Dutrieux Baraffe, Julien Bect, Emmanuel Vazquez, et al.. A Bayesian approach for the optimal integration of renewable energy sources in distribution networks over multi-year horizons. 26th International Conference \& Exhibition on Electricity Distribution (CIRED 2021), Sep 2021, Online (initially planned in Geneva), France. hal-03158700

\section{HAL Id: hal-03158700 \\ https://hal-centralesupelec.archives-ouvertes.fr/hal-03158700}

Submitted on 4 Mar 2021

HAL is a multi-disciplinary open access archive for the deposit and dissemination of scientific research documents, whether they are published or not. The documents may come from teaching and research institutions in France or abroad, or from public or private research centers.
L'archive ouverte pluridisciplinaire HAL, est destinée au dépôt et à la diffusion de documents scientifiques de niveau recherche, publiés ou non, émanant des établissements d'enseignement et de recherche français ou étrangers, des laboratoires publics ou privés. 


\title{
A BAYESIAN APPROACH FOR THE OPTIMAL INTEGRATION OF RENEWABLE ENERGY SOURCES IN DISTRIBUTION NETWORKS OVER MULTI-YEAR HORIZONS
}

\author{
Bruno Barracosa ${ }^{1,2, *}$, Juliette Morin ${ }^{1}$, Héloüse Dutrieux Baraffe ${ }^{1}$, \\ Julien Bect ${ }^{2}$, Emmanuel Vazquez ${ }^{2}$, Josselin Fournel ${ }^{1}$ \\ ${ }^{1}$ EDF R\&D, Economic and Technical Analysis of Energy Systems (EFESE), Palaiseau, France \\ ${ }^{2}$ Université Paris-Saclay, CNRS, CentraleSupélec, Laboratoire des Signaux et Systèmes (L2S), France \\ * bruno.tebbal-barracosa@edf.fr
}

Keywords: DISTRIBUTION NETWORK PLANNING, RENEWABLE ENERGY INTEGRATION, SIMULATION OPTIMISATION, MULTI-OBJECTIVE OPTIMISATION, BAYESIAN OPTIMISATION.

\begin{abstract}
We propose a method to optimise the planning strategy of an active distribution network. The problem is formulated as the search for the planning strategy parameters minimising antagonist objectives. These objectives are computed using a numerical simulator of the distribution networks and stochastic scenarios. Since simulations take a high amount of CPU time, we suggest using Bayesian optimisation algorithms, where the costs are modelled with Gaussian random processes. The main idea is to compute predictions of the costs and uncertainty intervals, which are then used to guide the optimisation algorithm. A case study illustrates the performance of the proposed method.
\end{abstract}

\section{Introduction}

Distribution network planning plays a fundamental role in the development of cost-effective distribution networks capable of efficiently integrating Renewable Energy Sources (RES). When RES integration increases, Distribution System Operators (DSOs) may face significant amounts of network reinforcement, with subsequent costs and implementation times, which might hinder the development of these sources [1].

Planning tools that consider "no-network" solutions have been proposed to find alternative planning strategies to integrate these sources optimally [2]. In this work, we use the simulation tool proposed in [3] to compute the sequence of RES integration solutions (network reinforcements combined with no-network solutions), depending on the considered DSO strategy. A scenario builder allows considering the uncertainty due to the variability of production and consumption profiles, and producers arrival (date, technology, installed power, location). If a new scenario is randomly generated for each simulation, as herein, the tool can be seen as a stochastic simulator. The average quality of a planning strategy can be assessed using statistical indicators such as the average Net Present Value (NPV) of total costs and the average rate of customers with quality issues over the scenarios.

Building on this tool, we propose a new approach for the integration of RES by identifying optimal parameters (e.g., level of RES curtailment, level of reactive power) of the DSO strategy. The difficulty is threefold: (1) we are dealing with a multi-objective optimisation problem, (2) the variance of the outputs is high, and (3) the computation time for one simulation is significant. As a result, we are in the case of a stochastic, multi-objective optimisation problem, with expensive-to-evaluate objective functions.

To tackle this challenge, we propose to use a Bayesian optimisation technique, where unknown objective functions are modelled with Gaussian Processes (GPs). The GPs then yield predictions of the stochastic simulator's outputs (without actually running it). In the end, the predictions are used to estimate the Pareto-optimal solutions, i.e., the solutions where one objective can not be improved without worsening another one. In this work, we consider the ParEGO algorithm [4], which is a Bayesian algorithm for deterministic multi-objective problems based on a scalarisation technique, and we extend it to deal with stochastic simulators.

The outline of the article is as follows. The use of stochastic simulators in the context of distribution network planning is introduced in Section 2. Section 3 presents Bayesian optimisation techniques for such simulators. Section 4 provides numerical experiments that illustrate the prediction accuracy gains and evaluation budget savings of the proposed approach. A final section presents the main conclusions.

\section{Using stochastic simulators for electricity distribution network planning}

In traditional network planning, DSOs often reinforce the network whenever a connection study identifies possible current or voltage limit violation risks. However, innovative planning 


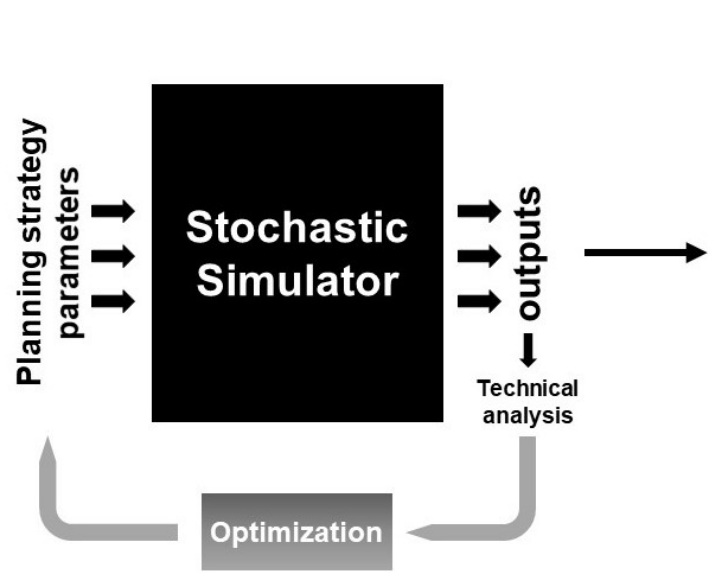

(a)

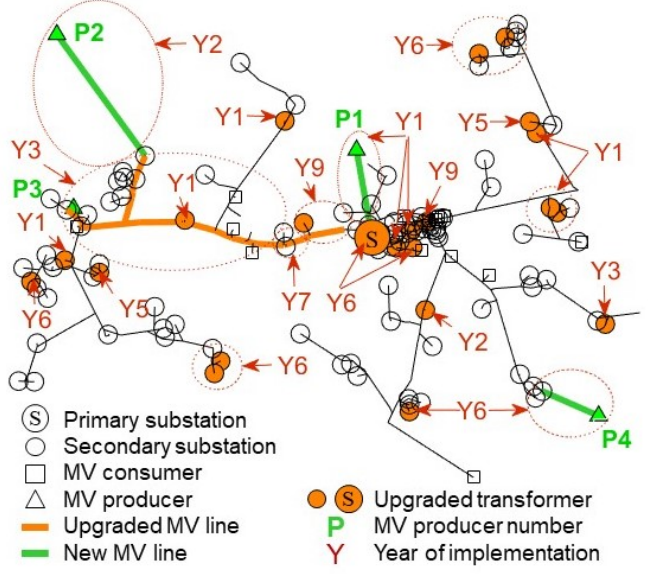

(b)

Fig. 1 (a) Illustration of the optimisation process of the input parameters of a stochastic simulator, and (b) example of resulting network planning decisions (reinforcements and new connections) for a given scenario and set of network planning parameters.

approaches attempt to find alternatives to reinforcement that could provide an interesting trade-off between cost reduction and acceptable risk level [5]. The aim is to identify the sequence of network reinforcements and alternative solutions, in a multi-year horizon, to minimise different outputs such as the overall cost [6].

To address this problem, Dutrieux et al. [3] proposed a simulation tool for the active distribution network planning, where the interactions between Medium-Voltage (MV) and LowVoltage (LV) networks are taken into account, and in which the DSO is assumed to not know in advance when RES will arrive on the network. By considering random scenarios on the producer's arrival (date, technology, installed power, location) and on the profiles of energy production and consumption, the simulator allows us to assess technical or economic outputs and their variability.

More precisely, for a given planning strategy (a set of planning rules), a given scenario, and an initial distribution network, the simulator sequentially emulates the DSO decisionmaking process to connect new RES and prevent and remove limit violations. This process uses a decision tree that has planning strategy parameters as inputs. Several outputs can then be extracted through technical or economic analysis, which can be fed to an algorithm to optimise the planning strategy. In this study, the selected outputs are the total cost NPV and the average annual rate of consumers with quality issues (both to be minimised). Figure 1 (a) illustrates the optimisation procedure and Figure 1 (b) presents an example of resulting network planning decisions (reinforcements and new connections).

We propose using this tool as a stochastic simulator by generating a new random scenario at each simulation run.

Several challenges must be considered in this approach:

(a) Since we are considering multiple objectives, the goal is not to identify a single optimal solution but rather a set of solutions that represent optimal trade-offs between the conflicting objectives. (b) When using a simulator, there is no analytic expression of the objective functions.

(c) Since the simulator is stochastic, statistical indicators must be estimated by using many simulation outputs.

(d) Each simulation takes about one minute. Thus, for example, 50.000 simulation results on $20 \mathrm{CPUs}$ take more than forty hours to obtain.

As the objective funtions are costly-to-evaluate, it is not possible to use traditional multi-objective optimisation where many evaluations are necessary to properly estimate the problem solutions (e.g., genetic algorithms). Additionally, the lack of analytical expression limits the use of techniques that rely on known properties of the objective function (e.g., convexity). Considering these constraints, the use of Bayesian optimisation techniques for stochastic simulators is introduced in the following section.

\section{Bayesian optimisation for stochastic simulators}

Bayesian optimisation has shown its usefulness when the functions to be optimised are expensive to evaluate. The main idea is to use a Gaussian Process (GP) to predict an unknown function from some of its deterministic or noisy evaluations, as illustrated in Figure 2. The use of a GP makes it possible to efficiently guide the optimisation process-see, e.g., [7] for a review or [8] for a tutorial. One of the most widely used algorithm for single-objective optimisation is the Efficient Global Optimisation (EGO) algorithm [9], which consists of choosing a sequence of evaluation points maximising the Expected Improvement (EI) sampling criterion.

In multi-objective optimisation, the objective is to identify the Pareto set of solutions, which corresponds to the set of points for which it is not possible to improve all objectives simultaneously. The Pareto front corresponds to the image, in the objective space, of the Pareto set. Bayesian approaches 


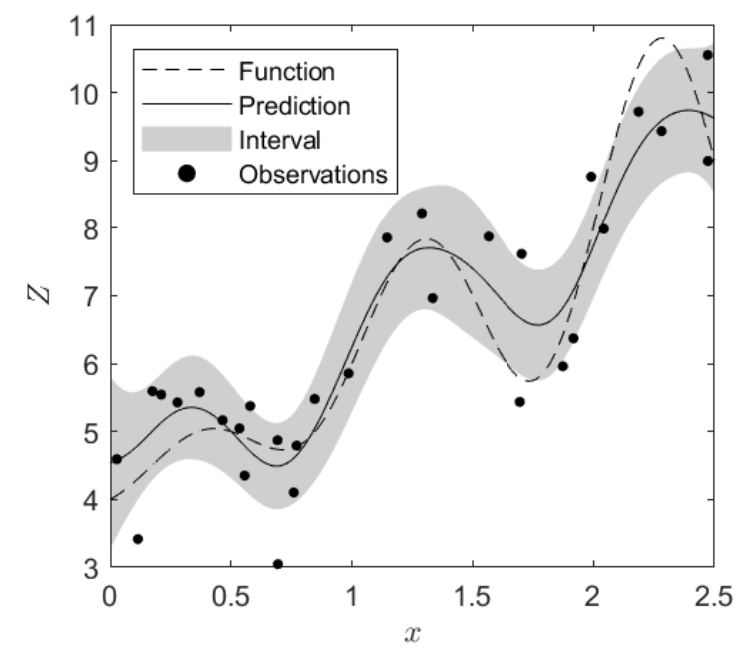

Fig. 2 Probabilistic model constructed from noisy observations (dots) of an unknown function (dashed line), its prediction of the unknown funtion (full line), and an prediction uncertainty interval (grey area).

for multi-objective optimisation have seen some important developments in the last few years (see, for instance [11-13]).

In this work, we focus on scalarisation techniques as proposed in the ParEGO algorithm [4], which solves deterministic multi-objective problems by iteratively solving several simpler single-objective problems. The main idea of the ParEGO algorithm is to use, as an objective function, an augmented Tchebycheff function

$$
\tilde{f}(x)=\max _{j}\left[\omega_{j} f_{j}(x)\right]+\rho \sum_{j} \omega_{j} f_{j}(x), \quad \rho>0,
$$

which aggregates the objective functions $f_{j}$ using random positive weights $\omega_{j}$.

In a deterministic setting, at each iteration $n$, the values of the objective functions $f_{j}$ at the previously evaluated points $X_{1}, \ldots, X_{n}$ are aggregated using (1). Then the ParEGO algorithm builds a GP model $\tilde{\xi}_{n}$ of $\tilde{f}$ using the aggregated values $\tilde{f}\left(X_{1}\right), \ldots, \tilde{f}\left(X_{n}\right)$ and, using the EI sampling criterion, selects a new evaluation point $X_{n+1}$. This point is expected to correspond to a small value of $\tilde{f}$ according to the GP model $\tilde{\xi}_{n}$. Since the minimum of a Tchebycheff function generally corresponds to a Pareto-optimal solution [10], it is possible to target the whole Pareto front when varying the weights randomly.

In our stochastic setting, we view the simulation results for a particular scenario as the sum of mean costs $f_{j}$ and random Gaussian perturbations. Our objective is to estimate the Pareto set of the mean cost functions $f_{j}$ from noisy evaluations of these costs. In this case, the EI sampling criterion is not a suitable choice because it considers as a reference the minimum among the previous noisy evaluations. Several criteria have been identified as suitable to the mono-objective noisy case-see, e.g., [14] for a review.

Here, we propose to extend ParEGO to the stochastic setting by replacing the EI sampling criterion with the Knowledge Gradient (KG) sampling criterion [15]. The main idea of $\mathrm{KG}$ is to select, as the new point to evaluate, the point that is expected to yield the smallest value for the minimum $\tilde{m}_{n+1}$ of the posterior mean of $\tilde{\xi}_{n+1}$ :

$$
X_{n+1}=\underset{x}{\arg \min } \mathrm{KG}_{n}(x),
$$

with

$$
\mathrm{KG}_{n}(x)=\mathbb{E}\left[\tilde{m}_{n+1} \mid X_{n+1}=x\right] .
$$

Then, a new simulation is performed at $X_{n+1}$. The process is repeated until a stopping criterion is met, for instance, when a prescribed budget of evaluations is exhausted.

The Pareto set can be estimated at each iteration using the GP models to generate predictions over the finite input space considered for this problem.

Compared with other existing methods for stochastic multiobjective optimisation $[11,16,17]$, ours has the advantage of simplicity.

\section{Numerical experiments}

We consider a French 390-bus network composed of one $90 / 20-\mathrm{kV}$ primary substation, four $20-\mathrm{kV}$ radial feeders, and 114 20/0.4-kV secondary substations (as in [3]). The peak load is about 9 MVA. At least $20 \mathrm{MW}$ of RES (wind and photovoltaic farms) are expected to request connection to this network in the coming ten years.

In our experiments, a simplified (faster-to-compute) emulator is used instead of the actual stochastic simulator, producing values equal to the average value of the real simulator with a zero-mean homoscedastic Gaussian white noise.

The objective is to minimise the average net present cost and the average rate of consumers with quality issues, by adequately selecting two decision variables: (1) the minimum $\tan \phi$ that the DSO can impose to each generator, and (2) the maximum annual generation curtailment rate allowed by each generator. Parameter $\tan \phi$ is allowed to range in the interval $[-0.6,0]$ and the curtailment rate ranges in $[0,0.1]$. We consider a regular search grid of size $21 \times 21$ for estimating the set of Pareto optimal solutions.

We start with an initial space-filling design of experiments of 20 points on the search grid. The design is constructed to maximise the minimum distance between any two points [18]. For each point of the initial design, ten evaluations of the simulator are performed.

Whenever GP models are mentioned, a Matérn-5/2 anisotropic covariance function is used with parameters estimated using the restricted maximum likelihood criterion. The STK toolbox [19], which has a ready-to-use Matlab implementation of the KG criterion, was used.

The models are used to generate estimations of the Pareto front and the Pareto set. The estimations are compared with the true sets using two metrics: the volume of the symmetric difference for the Pareto front, and the proportion of misclassified input points for the Pareto set.

Performance metrics are averaged over 500 runs and, for each, a budget of 50.000 simulator evaluations is considered.

We compare two different approaches: (1) a Monte-Carlo approach, where the next point to evaluate is chosen randomly, 
uniformly on the search space, and (2) a ParEGO + KG approach where the next point to evaluate is chosen as explained in Section 3. Whenever a point to evaluate is selected, a batch of 200 evaluations is performed at that point.

Figure 3 presents the performance metrics, showing that ParEGO+KG is more efficient than the Monte-Carlo approach (final metric result highlighted with a dashed line).
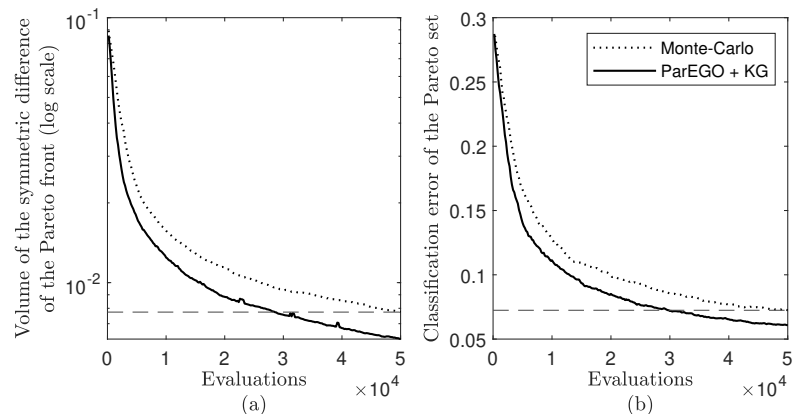

Fig. 3 Comparison of average performance metrics: (a) volume of the symmetric difference of the Pareto front, and (b) classification error of the Pareto set, over 500 experiments, for Monte-Carlo and ParEGO + KG approaches.

More precisely, considering the estimation of the Pareto front (Figure 3 (a)), the ParEGO+KG approach achieves on average the same performance level as the Monte-Carlo approach using only $57 \%$ of the evaluation budget. In other words, a $20 \%$ performance gain for the same simulation budget. Regarding the quality of the estimation of the Pareto set (Figure 3 (b)), the ParEGO+KG approach achieves, on average, the same performance as the Monte-Carlo approach using $60 \%$ of the evaluation budget, or a $12 \%$ performance gain for the same simulation budget.

Instead of considering an average result, let us now consider one among the 500 experiments performed using the ParEGO+KG approach. After using the evaluations budget, a GP model is fitted and used to generate estimates of the final Pareto front and Pareto set, as presented in Figure 4.

In Figure 4 (a), by generating estimations for all the input space points (light grey), a Pareto domination rule can be applied, and all the Pareto-optimal solutions can be identified (dark grey). These represent all the possible reasonable trade-offs among the two defined objectives and are all possible solutions to be chosen by the DSO.

Figure 4 (b) depicts the Pareto set estimation, i.e., all the points in the input space for which the representation in the objective space (Figure 4 (a)) is Pareto-optimal. When comparing our estimation with the true Pareto set (constructed from the known averages of our Gaussian simulator and represented with dots), it is possible to identify the good estimations (dark grey dots), the missed solutions (light grey dots), and bad estimations (red crosses).

When confronted with a set of possible solutions, a decisionmaker needs to select among competing objectives. Let us briefly compare three optimal solutions identified in the Pareto front and set in Figure 4 (see the numbered red circles). By looking at the Pareto front (Figure 4 (a)), it becomes clear that

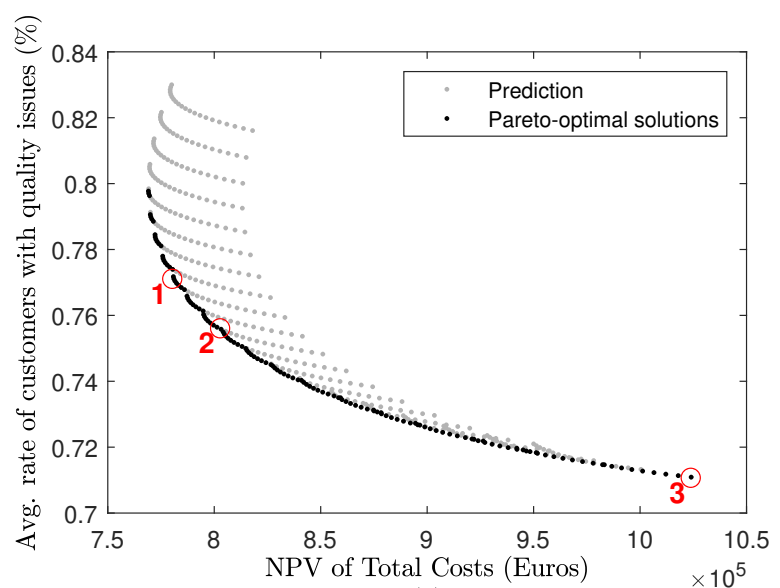

(a)

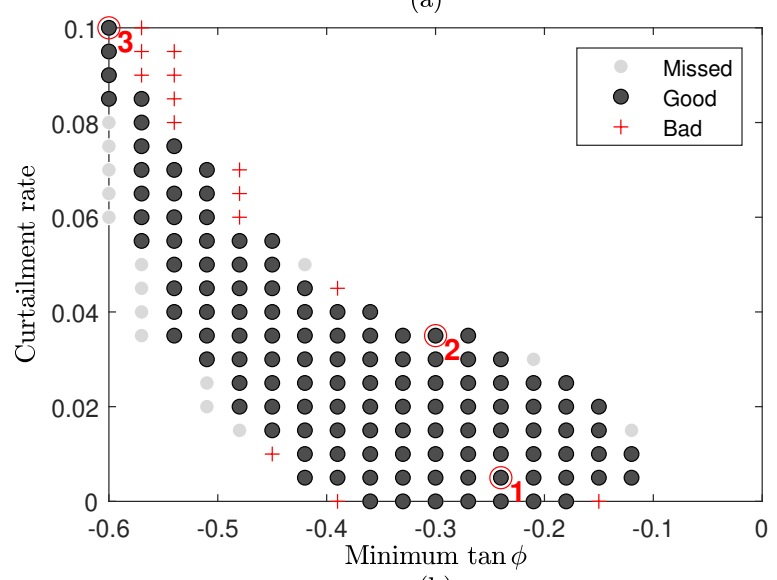

(b)

Fig. 4 (a) Pareto front, and (b) Pareto set estimation for a given optimisation run, using a ParEGO + KG approach, and a total budget of evaluations. Points 1, 2 and 3 represent solutions estimated to be Pareto-optimal.

solution 1 should be chosen if we favour cost reduction, while solution 3 if we are more sensitive to the quality of service. Solution 2 is one of many possible trade-offs between objectives. By looking at their location in the Pareto set (Figure 4 (b)), we can quickly identify the corresponding planning strategy parameters ( $\tan \phi$ and curtailment rate).

However, outputs have a stochastic nature, and the actual results of a planning strategy implementation depend on what happens in terms of producers arrival (date, technology, installed power, location) and energy production and consumption profiles. Figure 5 presents the simulation output distribution at each of the optimal solutions identified in Figure 4, and illustrates the diversity of possible outcomes.

There is no significant visual difference among the solutions concerning quality issues because of the high output variance (Figure 5 (b)). On the other hand, the chosen solution's impact on average cost distribution is more apparent (Figure 5 (a)).

Although the goal is to provide a set of Pareto-optimal solution to the decision-maker, it seems that output variability should play a relevant role in the final selection of the corresponding planning strategy parameters ( $\tan \phi$ and curtailment rate). By considering output variability, a better risk-reward ratio could be obtained from the decision-making process. 

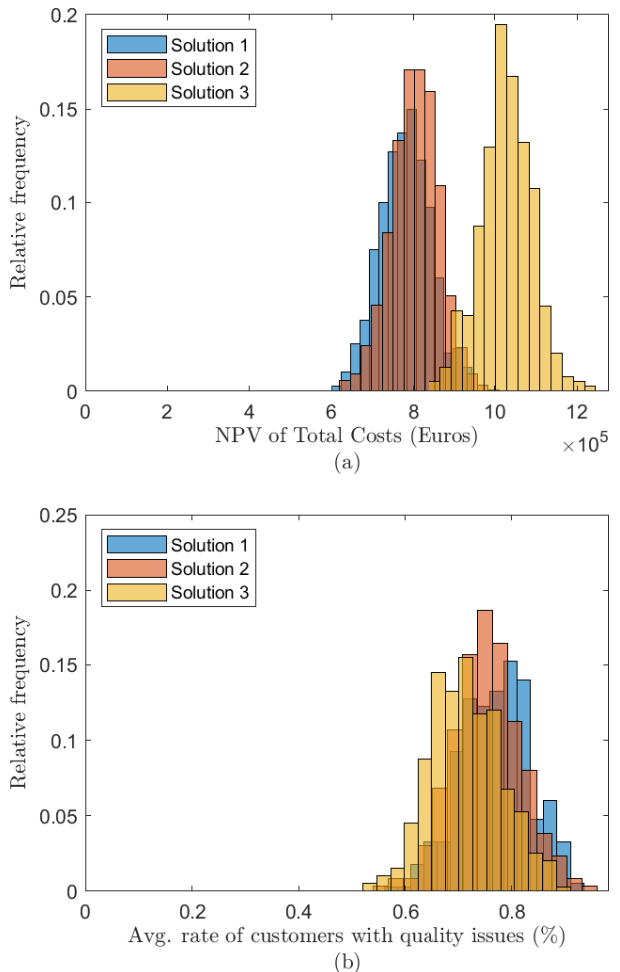

Fig. 5 Performed simulations output distribution for the two objectives: (a) total costs NPV, and (b) average rate of customers with quality issues, for the Pareto-optimal solutions identified by red circles in Figure 4.

\section{Conclusion}

This paper presents an approach for the optimisation of the parameters of a distribution network planning strategy using a stochastic simulator and Bayesian optimisation. It has been shown on a problem with two parameters and two antagonist objectives that the proposed approach reduces the number of simulations by as much as $40 \%$ compared to a Monte-Carlo approach.

Future work includes assessing the performance of the proposed approach when considering the industrial stochastic simulator and its tractability in case the number of parameters and/or the number of objectives increases.

\section{References}

[1] Delille, G., Malarange, G., and Gaudin, C.: 'Analysis of the options to reduce the integration costs of renewable generation in the distribution networks, part 2: a step towards advanced connection studies taking into account the alternatives to grid reinforcement', CIRED, Stockholm, Sweden, June 2013, paper 1356

[2] CIGRE Working Group C6.19, 'Planning and Optimization Methods for Active Distribution Systems' (CIGRE, 2014), pp. 1-184

[3] Dutrieux, H., Delille, G., François, B., et al.: 'An innovative method to assess solutions for integrating renewable generation into distribution networks over multi-year horizons', CIRED, Lyon, France, June 2015, paper 1103

[4] Knowles, J.: 'ParEGO: A hybrid algorithm with on-line landscape approximation for expensive multiobjective optimization problems', IEEE Transactions on Evolutionary, 2006, 10, (1), pp. 50-66

[5] Pilo, F., Celli, G., Ghiani, E., et al.: 'New electricity distribution network planning approaches for integrating renewable', WENE, 2013, 2, pp. 140-157

[6] Dutrieux, H., Delille, G., and Francois, B.: Assessing the impacts of distribution grid planning rules on the integration of renewable energy sources', IEEE Powertech, Eindhoven, Netherlands, June 2015, pp. 1-6

[7] Shahriari, B., Swersky, K., Wang, Z., et al.: 'Taking the human out of the loop: A review of Bayesian optimization', Proceedings of the IEEE, 2015, 104, (1), pp. 148-175

[8] Frazier, P.: 'A Tutorial on Bayesian Optimization', arXiv, 2018

[9] Jones, D. R., Schonlau, M., and Welch, W. J.: 'Efficient global optimization of expensive black-box functions', Journal of Global Optimization, 1998, 13, pp. 455-492

[10] Kasimbeyli, R., Ozturk, Z.K., Kasimbeyli, N., et al: 'Comparison of Some Scalarization Methods in Multiobjective Optimization', Bull. Malays. Math. Sci. Soc., 2019, 42, pp. 1875-1905

[11] Hunter, S. R., Applegate, E. A., Arora, V., et al.: 'An introduction to multiobjective simulation optimization', ACM Transactions on Modeling and Computer Simulation, 2019, 29, (1), pp. 1-36

[12] Feliot, P., Bect, J., and Vazquez, E.: 'A Bayesian approach to constrained single-and multi-objective optimization', Journal of Global Optimization, 2017, 67, pp. 97-133

[13] Rojas-Gonzalez, S., and Van Nieuwenhuyse, I.: 'A survey on kriging-based infill algorithms for multiobjective simulation optimization', Computers \& Operations Research, 2020, 116, pp. 104869

[14] Picheny, V., Wagner, T., and Ginsbourger, D.: 'A benchmark of kriging-based infill criteria for noisy optimization', Structural and Multidisciplinary Optimization, 2013, 48, pp. 607-626

[15] Frazier, P., Powell, W., and Dayanik, S: 'The knowledgegradient policy for correlated normal beliefs', INFORMS Journal on Computing, 2009, 21, (4), pp. 599-613

[16] Zuluaga, M., Sergent, G., Krause, A., et al.: 'Active learning for multi-objective optimization', Proceedings of the 30th International Conference on Machine Learning, Atlanta, USA, June 2013, pp. 462-470

[17] Gonzalez, S. R., Jalali, H., and Van Nieuwenhuyse, I.: 'A multiobjective stochastic simulation optimization algorithm', 2020, European Journal of Operational Research, 284, (1), pp. 212-226.

[18] Johnson, M. E., Moore, L. M., and Ylvisaker, D.: 'Minimax and maximin distance designs', Journal of Statistical Planning and Inference, 1990, 26, (2), pp. 131-148

[19] Bect, J., Vazquez, E., et al.: 'STK: a Small (Matlab /Octave) Toolbox for Kriging', Release 2.6, http: // kriging. sourceforge. net, 2019. 